\title{
Progress on MBE Grown Type-II Superlattice Photodiodes
}

\author{
C. J. Hill', J.V. Li, J.M. Mumolo and S.G. Gunapala \\ Jet Propulsion laboratory, California Institute of Technology, Pasadena, CA 91109 USA
}

\begin{abstract}
The closely lattice-matched material system of InAs, GaSb, and AlSb, commonly referred to as the $6.1 \AA$ material system, has emerged as a fertile ground for the development of new solid-state devices. The flexibility of the system in simultaneously permitting type-I, type-II staggered, and type-II broken-gap band alignments has been the basis for many novel, high-performance heterostructure devices in recent years, including the GaInSb/InAs type-II strained layer superlattice infrared detectors proposed by Smith and Mailhiot $\left[^{1}\right]$ in 1987. The type-II superlattice design promises optical properties comparable to $\mathrm{HgCdTe}$, better uniformity, reduced tunneling currents, suppressed Auger recombination, and normal incidence operation $\left[{ }^{2},{ }^{3}\right]$. In 1990, Chow and co-workers first reported $\mathrm{Ga}_{1-\mathrm{x}} \mathrm{In}_{\mathrm{x}} \mathrm{Sb} / \mathrm{InAs}$ superlattice materials with high structural quality, LWIR photoresponse, and LWIR photoluminescence $\left[{ }^{4}\right]$. Later, researchers demonstrated excellent detectivity (approaching $\mathrm{HgCdTe}, 8-\mu \mathrm{m}$ cutoff, $77 \mathrm{~K}$ ) on individual superlattice devices $\left.{ }^{5}\right]$. Currently, superlattice detector technology is undergoing the transition from single element detectors into high-performance focal plane imaging arrays $\left[{ }^{6}\right]$.

Here we report on the status of superlattice diodes grown and fabricated at the Jet Propulsion Laboratory designed for infrared absorption in the mid $(2-5 \mu \mathrm{m})$ and long wavelength $(8-12 \mu \mathrm{m})$ infrared ranges. Our mid-wavelength infrared devices display a zero bias differential resistance-area product as high as $1 \mathrm{e} 6 \mathrm{Ohmcm}^{2}$ at $80 \mathrm{~K}$ with a $5 \mu \mathrm{m}$ cutoff, with a corresponding detectivity of nearly $1 \mathrm{e} 13$ Jones. These detectors continue to function at room temperature with a detectivity of nearly $1 \mathrm{e} 9$ Jones. In the long wavelength region, we have produced devices with detectivities as high as $8 \times 10^{10}$ Jones with a differential resistance-area product greater than $6 \mathrm{Ohmcm}^{2}$ at $80 \mathrm{~K}$ with a long wavelength cutoff of approximately $12 \mu \mathrm{m}$. A typical IV curve a $12 \mu \mathrm{m}$ cutoff device is shown in Figure 1. Responsivity, detectivity and quantum efficiency for a typical $12 \mu \mathrm{m}$ cutoff device is shown in Figure 2. In addition to detector performance results, recent progress in epitaxial regrowth as a passivation method and progress on long-wavelength imaging array fabrication will be presented.
\end{abstract}

\footnotetext{
*Corresponding author: cory.j.hill@.jpl.nasa.gov, tel 818-393-7121 fax 818-393-4540
} 


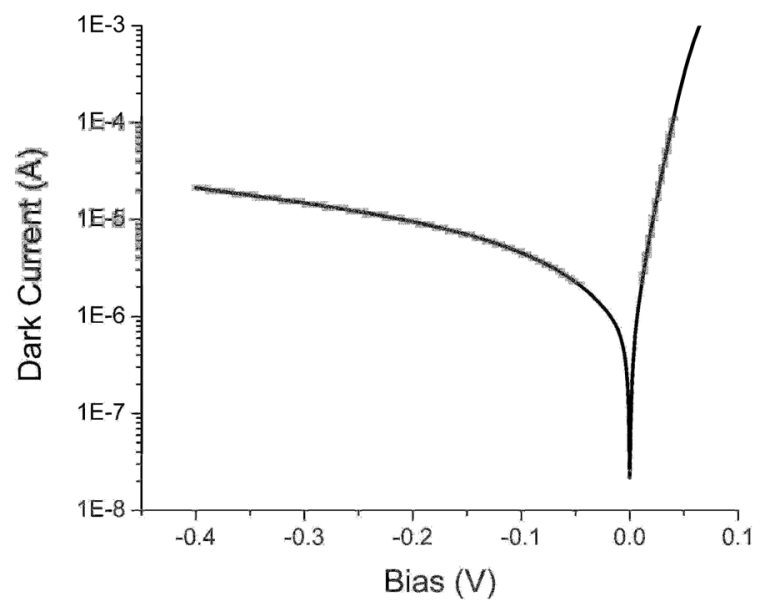

Figure 1. Dark current vs. applied bias for a typical $250 \times 250 \mu \mathrm{m}$ test device at $80 \mathrm{~K}(12 \mu \mathrm{m}$ cutoff devices).

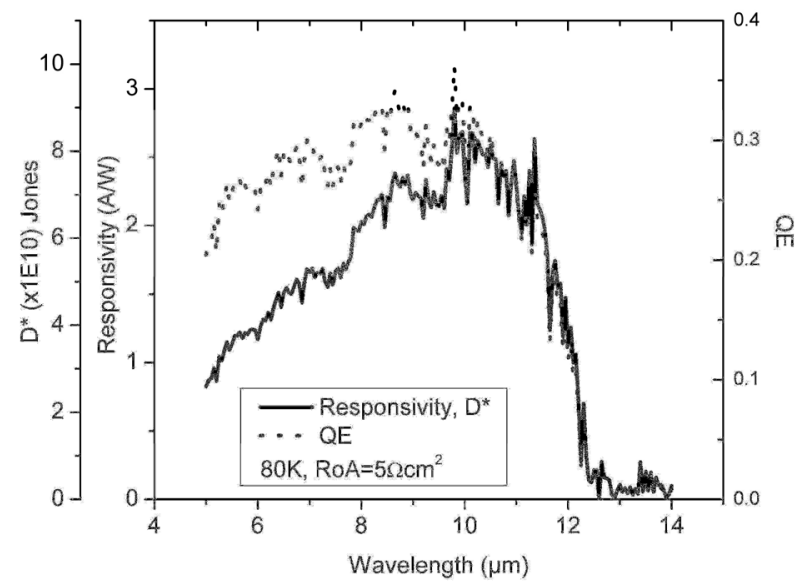

Figure 2. D*, responsivity, and QE (dotted, right axis) for the $12 \mu \mathrm{m}$ cutoff, large-area superlattice photodiodes.

${ }^{1}$ D.L.Smith and C.Mailhiot, J. Appl. Phys. 62, 2545 (1987).

${ }^{2}$ C.Mailhiot, and D.L.Smith, J. Vac. Sci. Technol. A 7, 445 (1989).

${ }^{3}$ R. H. Miles and D. H. Chow, in "Long Wavelength Infrared Detectors", edited by M. Razeghi, Chapter 7 (Gordon and Breach, Singapore, 1996); and references therein.

${ }^{4}$ R. H. Miles, D. H. Chow, J. N. Schulman, and T. C. McGill, Appl. Phys. Lett. 57, 801 (1990).

${ }^{5}$ F. Fuchs, U. Weimar, W. Pletschen, J. Schmitz, E. Ahlswede, M. Walther, J. Wagner, and P. Koidl, Appl. Phys. Lett. 71, 3251 (1997).

${ }^{6}$ M.Walther, R.Rehm, F.Fuchs, J. Schwitz, J.Fleissner, W.Cabanski, D.Eich, M.Finck, W.Rode, J.Wendler, R.Wollrab, and J.Ziegler, J. of Elect. Mater. 34 (6): p. $722-725$ (2005) 